\title{
Elastic scattering and rotational excitation of nitrogen molecules by sodium atoms
}

\section{Citation}

Loreau, Je\#rome, Peng Zhang, and Alexander Dalgarno. 2011. "Elastic Scattering and Rotational Excitation of Nitrogen Molecules by Sodium Atoms." The Journal of Chemical Physics 135, no. 17: 174301.

\section{Published Version}

doi:10.1063/1.3653983

\section{Permanent link}

http://nrs.harvard.edu/urn-3:HUL.InstRepos:12718787

\section{Terms of Use}

This article was downloaded from Harvard University's DASH repository, and is made available under the terms and conditions applicable to Other Posted Material, as set forth at http:// nrs.harvard.edu/urn-3:HUL.InstRepos:dash.current.terms-of-use\#LAA

\section{Share Your Story}

The Harvard community has made this article openly available.

Please share how this access benefits you. Submit a story.

\section{Accessibility}




\section{AIP chemicical Physics}

\section{Elastic scattering and rotational excitation of nitrogen molecules by sodium atoms}

Jérôme Loreau, Peng Zhang, and Alexander Dalgarno

Citation: J. Chem. Phys. 135, 174301 (2011); doi: 10.1063/1.3653983

View online: http://dx.doi.org/10.1063/1.3653983

View Table of Contents: http://jcp.aip.org/resource/1/JCPSA6/v135/i17

Published by the American Institute of Physics.

\section{Related Articles}

Relaxation of energetic S(1D) atoms in Xe gas: Comparison of ab initio calculations with experimental data J. Chem. Phys. 135, 024304 (2011)

Ultracold $\mathrm{O} 2+\mathrm{O} 2$ collisions in a magnetic field: On the role of the potential energy surface

J. Chem. Phys. 134, 124310 (2011)

Cold and ultracold $\mathrm{NH}-\mathrm{NH}$ collisions: The field-free case

J. Chem. Phys. 134, 124309 (2011)

Quantum and classical study of surface characterization by three-dimensional helium atom scattering J. Chem. Phys. 134, 024319 (2011)

Efficient numerical method for locating Feshbach resonances of ultracold molecules in external fields J. Chem. Phys. 134, 014101 (2011)

\section{Additional information on J. Chem. Phys.}

Journal Homepage: http://jcp.aip.org/

Journal Information: http://jcp.aip.org/about/about_the_journal

Top downloads: http://jcp.aip.org/features/most_downloaded

Information for Authors: http://jcp.aip.org/authors

\section{ADVERTISEMENT}

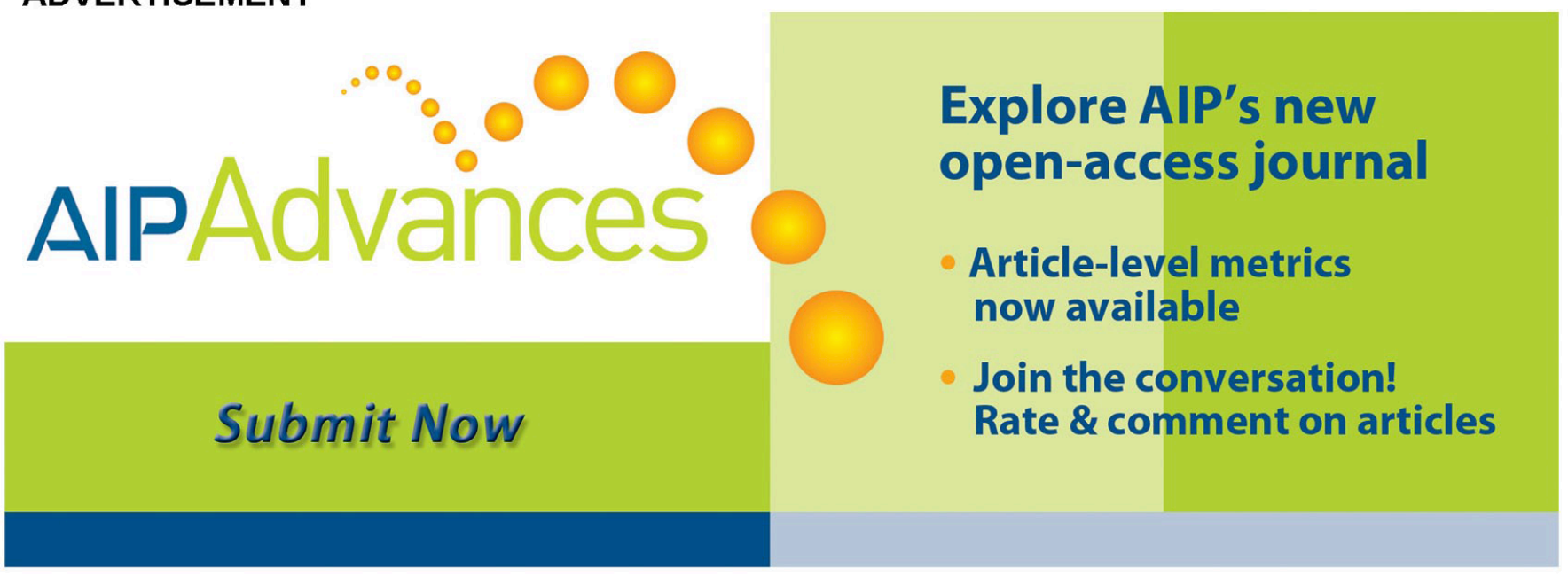




\title{
Elastic scattering and rotational excitation of nitrogen molecules by sodium atoms
}

\author{
Jérôme Loreau, ${ }^{\text {a) }}$ Peng Zhang, and Alexander Dalgarno \\ Institute for Theoretical Atomic, Molecular and Optical Physics (ITAMP), Harvard-Smithsonian \\ Center for Astrophysics, Cambridge, Massachusetts 02138, USA
}

(Received 11 July 2011; accepted 1 October 2011; published online 1 November 2011)

\begin{abstract}
A quantal study of the rotational excitation of nitrogen molecules by sodium atoms is carried out. We present the two-dimensional potential energy surface of the $\mathrm{NaN}_{2}$ complex, with the $\mathrm{N}_{2}$ molecule treated as a rigid rotor. The interaction potential is computed using the spin unrestricted coupledcluster method with single, double, and perturbative triple excitations (UCCSD(T)). The long-range part of the potential is constructed from the dynamic electric dipole polarizabilities of $\mathrm{Na}$ and $\mathrm{N}_{2}$. The total, differential, and momentum transfer cross sections for rotationally elastic and inelastic transitions are calculated using the close-coupling approach for energies between $5 \mathrm{~cm}^{-1}$ and $1500 \mathrm{~cm}^{-1}$. The collisional and momentum transfer rate coefficients are calculated for temperatures between $100 \mathrm{~K}$ and $300 \mathrm{~K}$, corresponding to the conditions under which $\mathrm{Na}-\mathrm{N}_{2}$ collisions occur in the mesosphere. () 2011 American Institute of Physics. [doi:10.1063/1.3653983]
\end{abstract}

\section{INTRODUCTION}

Collisions between sodium atoms and nitrogen molecules occurring in Earth's mesosphere play an important role in the understanding and development of sodium Laser Guide Stars (LGS). ${ }^{1}$ LGS are currently being developed at ground-based telescopes in an effort to improve the use of adaptive optics. This technique requires a reference source (a guide star) in order to efficiently correct the effects of the atmospheric distortion on the light received by telescopes. However, a sufficiently bright guide star is not always available in the region of the sky under observation. Sodium laser guide stars provide a solution to this problem by shining a laser at a wavelength of $589 \mathrm{~nm}$ into the atmosphere. This laser light is absorbed by sodium atoms present in the atmosphere and then reemitted, providing an artificial star that can be used as a reference star in any region of the sky. The process takes advantage of the natural sodium layer present in the mesosphere at an altitude of about $90 \mathrm{~km}$. This layer has a thickness of about $10 \mathrm{~km}$ and is mainly due to the burning of meteorites in the Earth's atmosphere so that sodium is produced continuously in the mesosphere. However, it is also removed by a series of complex mechanisms. At altitudes higher than $100 \mathrm{~km}$, sodium is still present as an ion (see Ref. 2 for a review on the chemistry of the sodium layer).

One of the main difficulties in the development of laser guide stars is to describe accurately the photon return from the mesosphere. Indeed, the LGS return flux depends on a combination of various effects and parameters (laser parameters, atmospheric parameters, telescope parameters, etc.). Holzlöhner $e t$ al. recently simulated the photon return flux by using the optical Bloch equations for the atomic density matrix. ${ }^{1}$ They emphasized the importance of including collisional processes between $\mathrm{Na}$ and $\mathrm{N}_{2}$ or $\mathrm{O}_{2}$ in the simulation, as they modify the velocity of sodium atoms and therefore the

\footnotetext{
a)Electronic mail: jloreau@cfa.harvard.edu.
}

Doppler shift of the atoms. In their modeling of the photon return flux, Holzlöhner et al. treated these collisions by assuming that the particles behave as hard spheres with an effective particle radius given by the Van der Waals radius.

The purpose of this work is to describe these $\mathrm{Na}-\mathrm{N}_{2}$ collisions using a fully quantal method. In addition to providing more precise values for the cross sections and rate constants, the differential cross sections obtained in this work can be used to model the velocity redistribution of the $\mathrm{Na}$ atoms.

In Sec. II, we calculate the potential energy surface of the ground state of the $\mathrm{Na}-\mathrm{N}_{2}$ Van der Waals complex using ab initio methods. We freeze the $\mathrm{N}-\mathrm{N}$ bond to its equilibrium distance, so that the vibration of $\mathrm{N}_{2}$ is neglected in the scattering calculations and the molecule is effectively treated as a rigid rotor. We compare our ab initio calculations with previous results obtained by Lee and Wright ${ }^{3}$ for the linear and T-shaped geometries. In Sec. III, we calculate the elastic and inelastic rotational cross sections, as well as the differential and momentum transfer cross sections, for energies in the range $5-1500 \mathrm{~cm}^{-1}$. We also calculate the corresponding rate constants for temperatures between $100 \mathrm{~K}$ and $300 \mathrm{~K}$.

\section{MOLECULAR DATA}

\section{A. Interaction potential}

The intermolecular potential between a sodium atom and a nitrogen molecule may be represented by three coordinates. We used the standard Jacobi coordinates $R, r, \theta$, where $R$ is the distance between the atom and the center of mass of the $\mathrm{N}_{2}$ molecule, $r$ denotes the length of the $\mathrm{N}-\mathrm{N}$ bond, and $\theta$ is the angle between $R$ and $r$. In this work, we have frozen the $\mathrm{N}_{2}$ internuclear distance to the equilibrium geometry, $r$ $=2.0743 a_{0}$. In Na- $\mathrm{N}_{2}$ collisions, this means that we neglect the vibrational motion of $\mathrm{N}_{2}$. This is appropriate since the ambient temperature in the sodium layer is about $T=185 \mathrm{~K}$, while the first excited vibrational state of $\mathrm{N}_{2}$ lies more than 
$2000 \mathrm{~cm}^{-1}$ above the ground vibrational state. If the nitrogen molecules are assumed to be in thermal equilibrium, only the $v=0$ state will be populated.

The ground state configuration of the sodium atom is $\left(1 s^{2} 2 s^{2} 2 p^{6} 3 s\right)^{2} S$, while the ground state of the nitrogen molecule is a ${ }^{1} \Sigma_{\mathrm{g}}^{+}$state. Therefore, the ground state of the $\mathrm{Na}-\mathrm{N}_{2}$ van der Waals complex corresponds to the ${ }^{2} A^{\prime}$ representation of the symmetry group $C_{s}$. For the particular geometries $\theta=0^{\circ}$ (linear) and $\theta=90^{\circ}$ (T-shaped), the ground state will be respectively a ${ }^{2} \Sigma^{+}$or a ${ }^{2} A_{1}$ state.

The Na- $\mathrm{N}_{2}$ complex was previously investigated by Lee and Wright ${ }^{3}$ for the linear and T-shaped geometries. They reported calculations using several $a b$ initio methods and basis sets, and concluded that the minimum of the $\mathrm{Na}-\mathrm{N}_{2}$ complex corresponds to the linear geometry. Using the RCCSD(T) method, they obtained a dissociation energy of $24 \mathrm{~cm}^{-1}$ and an equilibrium distance $R_{e} \sim 10.6$ a.u. However, the value of $R_{e}$ was strongly dependent on the method used. The T-shaped geometry was found to be a saddle point, lying $7.5 \mathrm{~cm}^{-1}$ higher than the linear minimum.

In the present study, the two-dimensional potential energy surface (PES) of the ground state was calculated using the spin unrestricted coupled-cluster method with single, double, and perturbative triple excitations (UCCSD(T)) (Refs. 4 and 5) implemented in the the MOLPRO 2009.1 package. ${ }^{6}$ We used the augmented polarized core-valence quadruple zeta (aug-cc-pCVQZ) basis set $^{7}$ to perform the $\mathrm{CC}$ calculations. The reference wave function employed in the CC calculations was generated from the spin restricted Hartree-Fock (ROHF) method. Nineteen electrons, including the $2 s^{2} 2 p^{6} 3 s$ electrons of the $\mathrm{Na}$ atom and the $2 s^{2} 2 p^{3}$ electrons of the $\mathrm{N}$ atoms, were correlated explicitly in the $\operatorname{UCCSD}(\mathrm{T})$ calculations. We constructed the PES using an uniform grid of 605 geometries. The grid for the intermolecular distance was $3 \leq R \leq 19.2 a_{0}$ with a step length of $0.3 a_{0}$, while for $\theta$ we used a grid $90^{\circ} \leq \theta \leq 180^{\circ}$ with a step size of $10^{\circ}$, with an additional series of points at $\theta=95^{\circ}$. The potential for $0^{\circ} \leq$ $\theta<90^{\circ}$ is obtained by symmetry with respect to $\theta=\pi / 2$. The effect of the basis set superposition error (BSSE) on the energy was taken into account by means of the counterpoise correction. $^{8}$

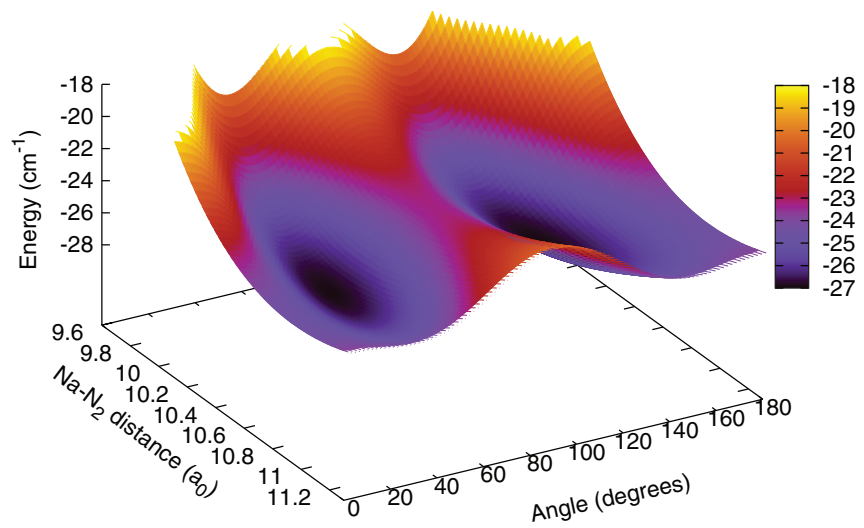

FIG. 1. Potential energy surface $V(R, \theta)$ for the $\mathrm{Na}-\mathrm{N}_{2}$ complex.
TABLE I. Geometry of the minimum of the $\mathrm{Na}-\mathrm{N}_{2}$ potential, as well as for the linear and T-shaped geometries (with $r$ fixed to the equilibrium geometry), dissociation energy $D_{e}$, and effect of the BSSE on $D_{e}$.

\begin{tabular}{lccc}
\hline \hline$R$ (a.u.) & $\theta$ (degrees) & $D_{e}\left(\mathrm{~cm}^{-1}\right)$ & $\operatorname{BSSE}\left(\mathrm{cm}^{-1}\right)$ \\
\hline 10.47 & 135 & 26.92 & 6.7 \\
10.46 & 90 & 23.24 & 6.1 \\
10.93 & 180 & 24.41 & 7.1 \\
\hline \hline
\end{tabular}

Our potential energy surface is shown in Figure 1. It is strongly repulsive at distances smaller than $9 a_{0}$, and weakly attractive for large $R$. The potential has a minimum at $\theta=135^{\circ}$ (and $\theta=45^{\circ}$ ) and the equilibrium distance is $R=10.47 a_{0}$, with a dissociation energy $D_{e}=26.92 \mathrm{~cm}^{-1}$. For the specific value $\theta=90^{\circ}$, the potential has a saddle point, with a dissociation energy of $23.24 \mathrm{~cm}^{-1}$ (lying only $3.7 \mathrm{~cm}^{-1}$ above the minimum), while for the linear geometry the dissociation energy is $24.41 \mathrm{~cm}^{-1}$. However, the minimum for the linear geometry occurs at a larger $\mathrm{Na}-\mathrm{N}_{2}$ distance. These results are summarized in Table I. The effect of the BSSE on the dissociation energies is also reported in the same table. For such a weakly bound complex, it is clear that the BSSE cannot be neglected as it can contribute significantly to the dissociation energy. We found that the effect of the BSSE on $D_{e}$ was $\sim 7 \mathrm{~cm}^{-1}$, or about $28 \%$ of the dissociation energy, a value similar to the one reported by Lee and Wright. For the linear geometry, the values of the dissociation energy and of the equilibrium distance are seen to be very close to the ones reported in Ref. 3 ( $D_{e}=24 \mathrm{~cm}^{-1}$ and $R_{e}=10.6$ a.u.). However, this geometry does not correspond to the global minimum of the potential. The T-shaped structure is confirmed to be a saddle point, although the energy gap between the minimum of this structure and the minimum of the linear structure is only of about $1 \mathrm{~cm}^{-1}$, as opposed to the value of $7.5 \mathrm{~cm}^{-1}$ reported in Ref. 3.

\section{B. Long-range interactions-Asymptotic potential}

The asymptotic long-range attractive potential $V_{\mathrm{as}}$ is dominated by the dispersion forces and can be constructed with an analytical form. We consider here only the leading term,

$$
V_{\mathrm{as}}=-\frac{C_{6}}{R^{6}},
$$

since the next term in the series, $C_{8} / R^{8}$, is not expected to contribute significantly.

In the case of the interaction between an $S$-state atom and a linear molecule, the $C_{6}$ coefficient is given by the sum of an isotropic and an anisotropic component: ${ }^{9}$

$$
C_{6}=C_{6,0} P_{0}(\cos \theta)+C_{6,2} P_{2}(\cos \theta),
$$

where $P_{0}$ and $P_{2}$ are the Legendre polynomials of order 0 and 2 , respectively. The isotropic and anisotropic dispersion coefficients may be derived by

$$
C_{6,0}=\frac{3}{\pi} \int_{0}^{\infty} \alpha^{\mathrm{Na}}(i \omega) \bar{\alpha}^{\mathrm{N}_{2}}(i \omega) d \omega,
$$




$$
C_{6,2}=\frac{1}{\pi} \int_{0}^{\infty} \alpha^{\mathrm{Na}}(i \omega)\left[\alpha_{\|}^{\mathrm{N}_{2}}(i \omega)-\alpha_{\perp}^{\mathrm{N}_{2}}(i \omega)\right] d \omega
$$

where $\alpha_{\|}(i \omega)$ and $\alpha_{\perp}(i \omega)$ are respectively the longitudinal and transverse dynamical electric dipole polarizabilities, evaluated as functions of imaginary frequencies $i \omega . \bar{\alpha}$ is the average dipole polarizability:

$$
\bar{\alpha}=\frac{1}{3}\left(\alpha_{\|}+2 \alpha_{\perp}\right) .
$$

The expressions (3) and (4) can be calculated efficiently using Gaussian quadrature. ${ }^{10}$ The integral is then replaced by a sum over $N$ quadrature points $\omega_{k}$, with weights $w_{k}$. The dynamic polarizabilities of the $\mathrm{N}_{2}$ molecule were evaluated using the time-independent linear response coupled cluster theory ${ }^{11}$ at the third-order in the fluctuation potential as implemented in MOLPRO. A triply augmented correlation consistent polarized core-valence sextuple zeta (t-aug-cc-pCV6Z) basis ${ }^{12}$ was employed in the coupled cluster calculations. Our computed static polarizabilities are $\alpha_{\perp}^{\mathrm{N}_{2}}=10.13$ a.u. and $\alpha_{\|}^{\mathrm{N}_{2}}=14.75$ a.u., which are consistent with other theoretical values in the ranges of 9.8-10.34 a.u. and 14.5-15.02 a.u., respectively. ${ }^{13}$

For $\mathrm{Na}$, we adopted the accurate many-body relativistic values reported in Ref. 14. The integration was realized using a 50-points Gaussian quadrature. We found $C_{6,0}$ $=206.70$ a.u. and $C_{6,2}=26.44$ a.u.. The accuracy of the derived dispersion coefficient can be further verified through the direct comparison with the UCCSD(T) calculations. Substituting the values of the $C_{6}$ coefficients into Eq. (1), the energy difference between the UCCSD(T) calculations and the asymptotic potential (1) at the last point of our computed grid $\left(R=19.2 a_{0}\right)$ is smaller than $0.2 \mathrm{~cm}^{-1}$ and depends on the value of $\theta$. In order to smoothly connect the ab initio points and the asymptotic potential, we used a switching function expressed as the product of a $R$-dependent and a $\theta$-dependent function. It is given by

$$
f(R, \theta)=-(1+\tanh (a R+b)) \cos (c(\theta+d)) .
$$

The four parameters $a, b, c, d$ were optimized with respect to the $a b$ initio points at large $R$ using a nonlinear least-squares Marquardt-Levenberg algorithm and are given in Table II. The root mean square deviation was $0.015 \mathrm{~cm}^{-1}$.

\section{SCATTERING CALCULATIONS}

\section{A. Method}

We calculated the cross sections for $\mathrm{Na}-\mathrm{N}_{2}$ collisions using the quantum close-coupling method of Arthurs and Dalgarno. ${ }^{15} \mathrm{~N}_{2}$ was treated in the rigid-rotor approximation being held fixed at its equilibrium bond-length. We will only

TABLE II. Parameters of the switching function (6).

\begin{tabular}{lcccc}
\hline \hline$a$ & $b$ & $c$ & $d$ \\
\hline-0.0478069 & 1.24173 & 0.171625 & 13.6938 \\
\hline \hline
\end{tabular}

briefly review the method here, as it has been described in detail in the literature. ${ }^{15,16}$

The Hamiltonian for the $\mathrm{Na}-\mathrm{N}_{2}$ complex in the rigid rotor approximation can be written as

$$
H=-\frac{1}{2 \mu} \nabla_{R}^{2}+H_{\mathrm{rot}}+V(R, \theta)
$$

where $\mu$ is the reduced mass of the $\mathrm{Na}-\mathrm{N}_{2}$ system, $V(R, \theta)$ is the interaction potential between $\mathrm{Na}$ and $\mathrm{N}_{2}$, and $H_{\text {rot }}$ describes the rotation of the $\mathrm{N}_{2}$ molecule. Its eigenvalues are the rotational energies,

$$
H_{\mathrm{rot}} Y_{j m_{j}}=\epsilon_{j} Y_{j m_{j}},
$$

where $j$ is the rotational quantum number, $m_{j}$ is its projection on the space-fixed $z$ axis, and $Y_{j m_{j}}$ are the spherical harmonics. In this work, we have represented the energy of the rotational levels of the nitrogen ${ }^{1} \Sigma_{\mathrm{g}}^{+}$ground state by

$$
\epsilon_{j}=\left(B_{e}-\alpha_{e} / 2\right) j(j+1)-D_{e} j^{2}(j+1)^{2},
$$

with the rotational constants of $\mathrm{N}_{2}$ given by ${ }^{17,18}$

$$
\begin{aligned}
& B_{e}=1.99824 \mathrm{~cm}^{-1}, \quad D_{e}=5.76 \times 10^{-6} \mathrm{~cm}^{-1}, \\
& \alpha_{e}=0.017318 \mathrm{~cm}^{-1} .
\end{aligned}
$$

In the close-coupling method, the total wave function of the $\mathrm{Na}-\mathrm{N}_{2}$ complex is expanded as a sum of products of radial and rotational functions. These basis channels functions are labelled by the rotational quantum number $j$ and by the orbital quantum number $l$. This expansion is then inserted in the Schrödinger equation with the Hamiltonian (7), leading to a set of second-order coupled differential equations for the radial functions which must be solved with appropriate boundary conditions. In these equations, the coupling between the different channels is represented by matrix elements of the intermolecular potential.

It is convenient to introduce the total angular momentum $\mathbf{J}=\mathbf{j}+\mathbf{l}$, where $\mathbf{j}$ is the angular momentum of $\mathrm{N}_{2}$ and $\mathbf{l}$ is the orbital momentum. The total angular momentum is conserved during the collision, so that the coupled equations are block-diagonal in $J$. The $S$ matrix elements $S_{j^{\prime} l^{\prime} j l}^{J}$ can be obtained from the asymptotic behavior of the radial functions. The cross section for the transition from an initial state $j$ to a final state $j^{\prime}$ is then given in terms of the $S$ matrix elements as

$\sigma_{j j^{\prime}}=\frac{\pi}{(2 j+1) k_{j}^{2}} \sum_{J=0}^{\infty} \sum_{l=|J-j|}^{|J+j|} \sum_{l^{\prime}=\left|J-j^{\prime}\right|}^{\left|J+j^{\prime}\right|}(2 J+1)\left|\delta_{j j^{\prime}} \delta_{l l^{\prime}}-S_{j^{\prime} l^{\prime} j l}^{J}\right|^{2}$,

where $k_{j}=\sqrt{2 \mu\left(E-\epsilon_{j}\right)}$ is the wavenumber in the entrance channel and $E$ is the total (kinetic plus rotor) energy.

We carried out the scattering calculations for collision energies between 5 and $1500 \mathrm{~cm}^{-1}$ using the nonreactive scattering code MOLSCAT of Hutson and Green. ${ }^{19}$ The radial equations were solved using the log-derivative propagator method. The log-derivative matrix is propagated on a grid of intermolecular distances between $R_{\min }$ and $R_{\max }$. At $R_{\max }$, the numerical calculations are matched with the asymptotic solutions and the $S$ matrix elements are extracted. We used a grid starting at $R_{\min }=3$ a.u., while the $R_{\max }$ was in the range 


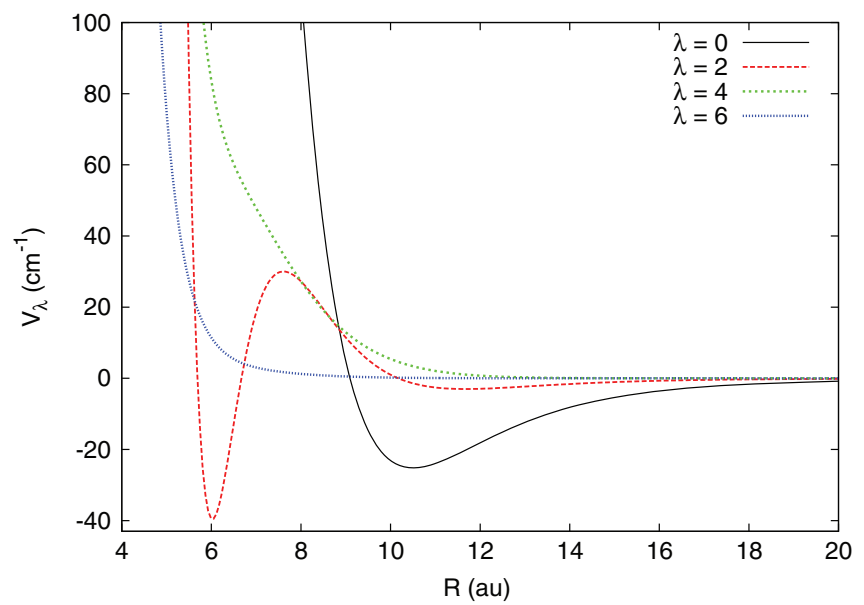

FIG. 2. The first four radial functions $V_{\lambda}(R)$ in the expansion (11) of the intermolecular potential.

50-150 a.u., depending on the energy of collision. For a particular energy, the propagation is carried out until convergence of the sum over the total angular quantum number $J$ in Eq. (10) is achieved. For the elastic cross sections, the number of partial waves required ranged from 50 for $E=5 \mathrm{~cm}^{-1}$ to about 400 for an energy of $1500 \mathrm{~cm}^{-1}$, while the convergence is faster for inelastic cross sections. At low energies ( $E \sim 10 \mathrm{~cm}^{-1}$ ), we found that it was typically necessary to include 5-6 closed channels in our calculations, while at high energy $\left(E \sim 1000 \mathrm{~cm}^{-1}\right)$ the inclusion of 2 closed channels was enough to guarantee convergence of the cross sections. At the highest energy considered in this work, this requires a basis set of about 30 rotational functions.

The calculation of the cross section is considerably simplified if the intermolecular potential $V(R, \theta)$ is expanded in terms of Legendre polynomials of order $\lambda$,

$$
V(R, \theta)=\sum_{\lambda} V_{\lambda}(R) P_{\lambda}(\cos \theta),
$$

where the $V_{\lambda}(R)$ are known as "radial strength functions." This equation can be inverted as

$$
V_{\lambda}(R)=(\lambda+1 / 2) \int_{0}^{\pi} V(R, \theta) P_{\lambda}(\cos \theta) \sin \theta d \theta,
$$

and evaluated using a Gauss-Legendre quadrature. Note that in the case of a collision between an atom and an homonuclear diatomic molecule, only even values of $\lambda$ appear in Eq. (11) due to the symmetry of the intermolecular potential. The first four radial functions $V_{\lambda}(R)$ are shown in Figure 2. In our calculations we found that it was sufficient to include terms up to $\lambda=10$ to ensure convergence of the cross sections.

In the modeling of LGS, one of the key quantities is the momentum transfer between $\mathrm{Na}$ and $\mathrm{N}_{2}$. The momentumtransfer cross section $\sigma_{j j^{\prime}}^{\mathrm{tr}}$, also called transport cross section, from an initial level $j$ to a final level $j^{\prime}$ may be obtained from the differential cross section as 20

$$
\sigma_{j j^{\prime}}^{\operatorname{tr}}\left(E_{c}\right)=2 \pi \int d \gamma \sin \gamma \frac{d \sigma_{j j^{\prime}}\left(E_{c}, \gamma\right)}{d \Omega} \Phi\left(E_{c}, \gamma\right),
$$

with the function

$$
\Phi\left(E_{c}, \gamma\right)=1-\sqrt{E_{c}^{\prime} / E_{c}} \cos \gamma,
$$

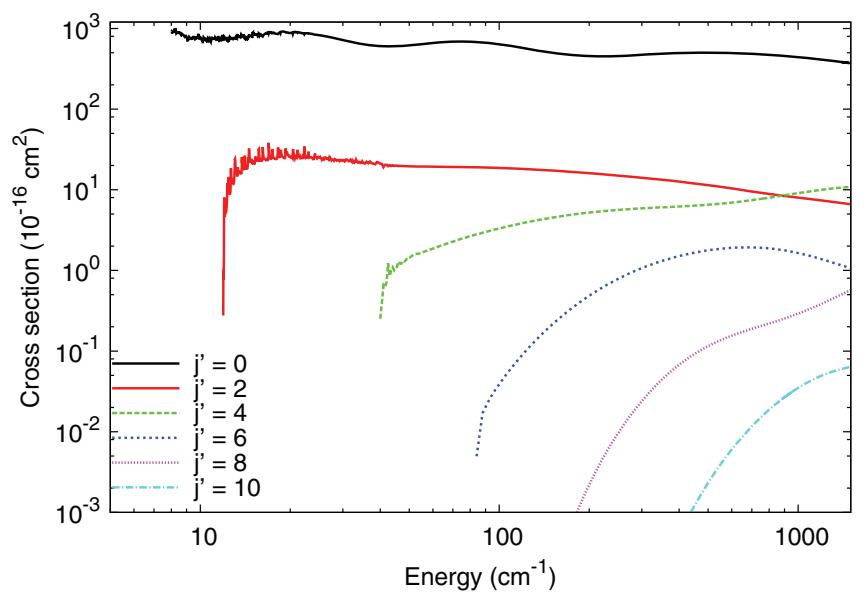

FIG. 3. Elastic and inelastic cross sections from the initial rotational level $j=0$ to the final rotational level $j^{\prime}$.

where $E_{c}^{\prime}$ is the final kinetic energy, related to the initial kinetic energy by

$$
E_{c}^{\prime}=E_{c}+\epsilon_{j}-\epsilon_{j^{\prime}}
$$

\section{B. Results and discussions}

\section{Cross sections}

The elastic and inelastic cross sections for transitions between the initial level $j=0$ and the final levels $j^{\prime}=0-10$ are shown in Figure 3. We observe that the collision is dominated by the elastic process at all energies, the cross section $j=0$ $\rightarrow j^{\prime}=0$ being almost two orders of magnitude larger than any of the inelastic cross sections. At energies below $1000 \mathrm{~cm}^{-1}$, the rotationally inelastic cross sections decrease with increasing final rotational level $j^{\prime}$. However, at an energy of about $1000 \mathrm{~cm}^{-1}$, the $0-4$ transition becomes the leading inelastic transition.

The cross sections can be understood qualitatively with the potential expansion (11). The leading term is the isotropic potential $V_{0}$, which drives the elastic transitions. At intermolecular distances smaller than 9 a.u., this term is much larger than the anisotropic terms, so that the collision can be expected to be mostly elastic. The radial functions $V_{\lambda}$ get smaller when $\lambda$ increases, except for the $V_{4}$ term which is larger than $V_{2}$ in the classically allowed region. The cross sections for transitions $\Delta j \geq 4$ thus quickly become negligible as $\Delta j=\left|j-j^{\prime}\right|$ increases, and there is a strong hierarchy between the inelastic cross sections.

At energies below $40 \mathrm{~cm}^{-1}$, the cross sections $0-0,0-2$, and $0-4$ exhibit an oscillatory behavior characteristic of resonances. These resonances at low kinetic energy are a consequence of the attractive potential well, which allows $\mathrm{Na}$ to be temporarily trapped and hence quasi-bound states to be formed before the molecular complex dissociates. The quasibound states may arise from the tunneling from the centrifugal barrier and from excitation of $\mathrm{N}_{2}$ to an asymptotically closed channel where $\mathrm{N}_{2}$ becomes temporarily trapped in one of the bound states of the potential well. ${ }^{21-23}$ Compared to the depth 


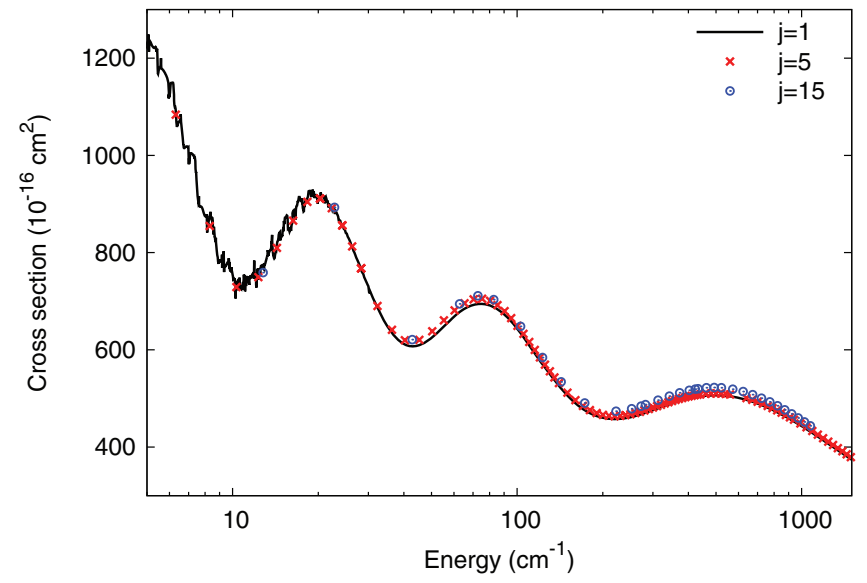

FIG. 4. Elastic cross sections for different values of $j$. Full line: $j=1$, crosses: $j=5$, and circles: $j=15$.

of the potential well, the energy spacing of the $\mathrm{N}_{2}$ rotational structure is small, so that both types of quasibound states occur in the same energy range.

The disappearance of the resonances as a function of the collision energy can be qualitatively understood by examining the radial functions $V_{\lambda}(R)$ : the spherical term $V_{0}$, which governs the elastic transitions, has a potential well of about $25.2 \mathrm{~cm}^{-1}$ positioned at $R=10.50$ a.u., close to the equilibrium distance of the van der Waals complex. The presence of this well explains the resonances found in the elastic cross sections at energies below $25 \mathrm{~cm}^{-1}$. The $V_{2}$ term, which dominates the $\Delta j=2$ transitions, also has a potential well, located around an intermolecular distance of 6 a.u., with a depth of $39.6 \mathrm{~cm}^{-1}$. However, the region $R<8$ a.u. is classically forbidden at low energy and the resonances can therefore be attributed to quasi-bound levels of the $\mathrm{Na}-\mathrm{N}_{2}$ complex. It should be noted that the resonances are gradually suppressed with increase in the initial rotational level, as can be observed on Figure 5. The shallow Van der Waals potential well supports only a limited number of excited quasi-bound levels, and levels corresponding to higher $j$ become virtual states producing no shape resonances, so that we observe no resonances for initial diatomic rotational levels $j \geq 2$.

In Figure 4, we show the elastic cross section for the rotational state $j=1$. In the energy range considered in this work, the general behavior of the elastic cross section is a decrease with increasing energy. However, the decrease is not monotonic and we observe three peaks located around $E_{c}$ $=20 \mathrm{~cm}^{-1}, E_{c}=75 \mathrm{~cm}^{-1}$, and $E_{c}=500 \mathrm{~cm}^{-1}$. On the same figure, we also display the elastic cross section for $j$ $=5$ (the most populated rotational level at $T=185 \mathrm{~K}$ ) and for the highly excited state $j=15$. Except for the presence of resonances at low energy for $j=1$, we see that the elastic cross section is almost independent of $j$. This peak structure is characteristic of glory undulations, as manifested by the plot of $\sigma_{e l} v^{2 / 5}$ vs $1 / v{ }^{24}$ The independence of the peak positions with respect to the initial rotational state indicates that the rotational time of $\mathrm{N}_{2}$ is short compared to a characteristic translational time and hence inelastic processes make little contribution to the glory undulations. A crude estimate shows that the rotational time of $\mathrm{N}_{2}$ is $\sim 10^{-13} \mathrm{~s}$ depending on the

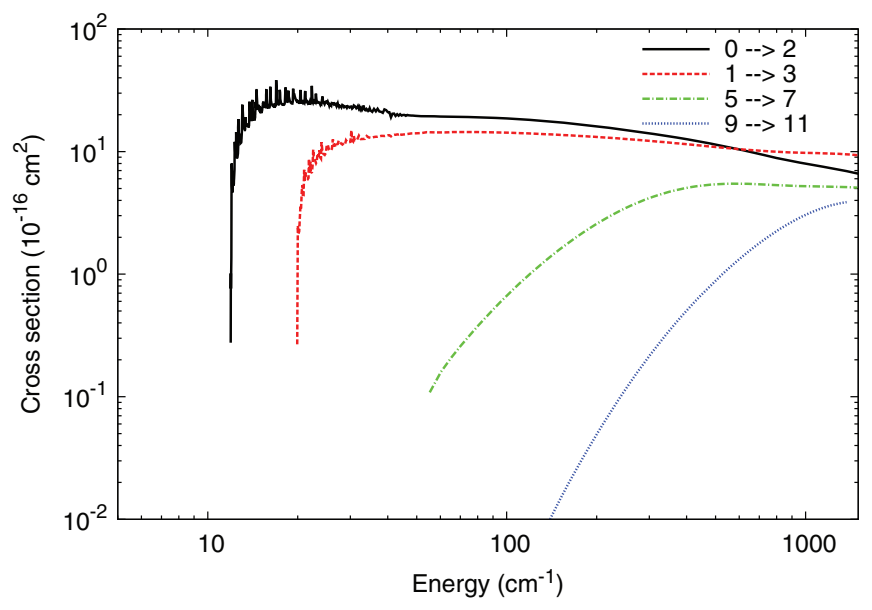

FIG. 5. Inelastic cross sections for the transition $j \rightarrow j+2$ for several values of $j$.

rotational level, and the translational time is estimated to be $\sim 10^{-12} \mathrm{~s}$ if one assumes a path length of about $15 a_{0}$. In the energy range of the present study, the inelasticity of scattering, defined as the ratio between the total inelastic cross section and the total cross section, is small, varying from $2 \%$ to $15 \%$ depending on the initial rotational state. The similarity of the elastic cross sections reflects the fact that the total cross section is independent of the initial state apart from the factor accounting for the detailed balance. ${ }^{25}$

The cross sections for rotational excitation with $\Delta j=2$ and $\Delta j=6$, respectively, from the initial rotational levels $j=0,1,5$ and $j=9$ are shown in Figures 5 and 6. By comparing the two figures, we see that as was observed for $j=0$, the inelastic cross sections are much smaller for $\Delta j=6$ than for $\Delta j=2$. Moreover, the inelastic cross sections decrease as the initial rotational level $j$ increases, although this observation is partly masked in Figures 5 and 6 due to threshold effects. Therefore, as $j$ increases, the collisions will be more and more dominated by the elastic process.

The differential cross sections are useful for the evaluation of momentum transfer in collisions of $\mathrm{Na}$ and $\mathrm{N}_{2}$.

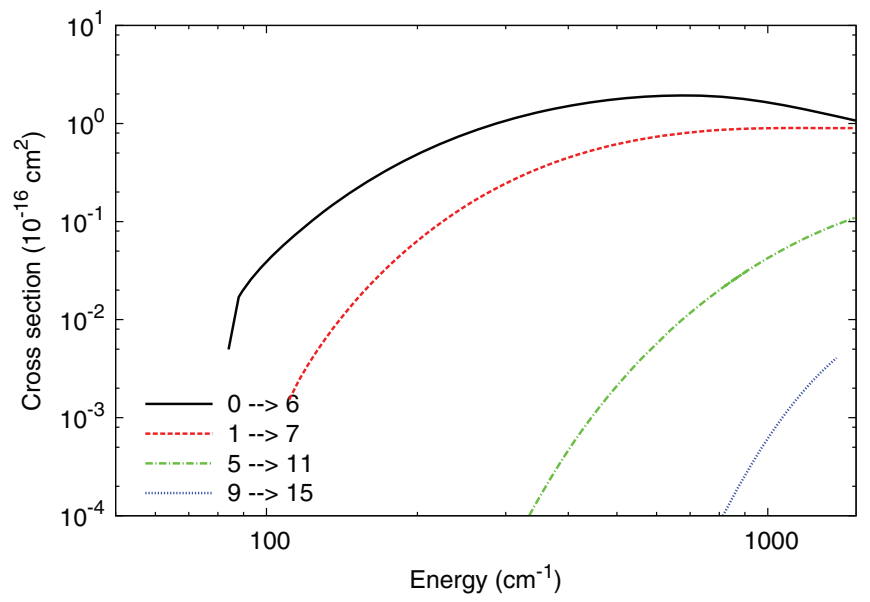

FIG. 6. Inelastic cross sections for the transition $j \rightarrow j+6$ for several values of $j$. 


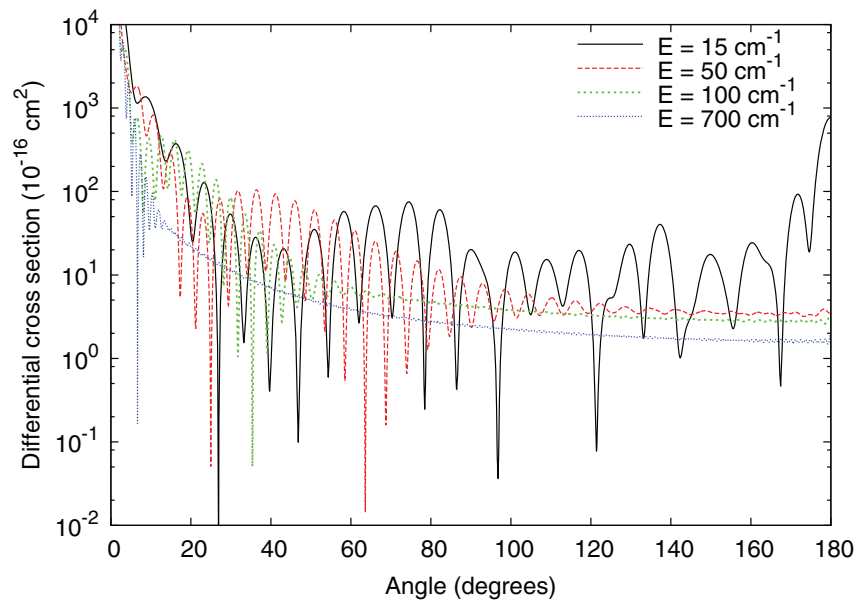

FIG. 7. Elastic differential cross section for $j=0$ for several energies, as a function of the scattering angle.

The elastic differential cross section for $j=0$ is shown in Figure 7 for various energies of collision. The differential cross sections present a fast oscillatory behavior as a function of the scattering angle $\gamma$ which reflects quantum mechanical interferences. At low energy, these oscillations occur at all angles, so that there is an important contribution from backward scattering. However, this contribution quickly decreases with increasing energy and the elastic collision becomes dominated by forward scattering. The inelastic cross section $j=0$ $\rightarrow j=2$ is presented in Figure 8 for a few selected energies. Similarly to the elastic cross section, we observe fast oscillations as a function of the scattering angle and a backward scattering contribution which decreases with increasing energy. However, the magnitude of these oscillations is much smaller.

The differential cross sections $d \sigma_{j j^{\prime}} / d \Omega$ are used to compute the momentum transfer cross section, given by Eq. (13). The total momentum transfer cross section, obtained by summing $\sigma_{j j^{\prime}}^{\mathrm{tr}}$ over all the final rotational states $j^{\prime}$, is shown in Figure 9 for $j=1$. We also display on the same figure the contribution of the elastic and inelastic scattering. At low energy, the momentum transfer cross section is entirely due to

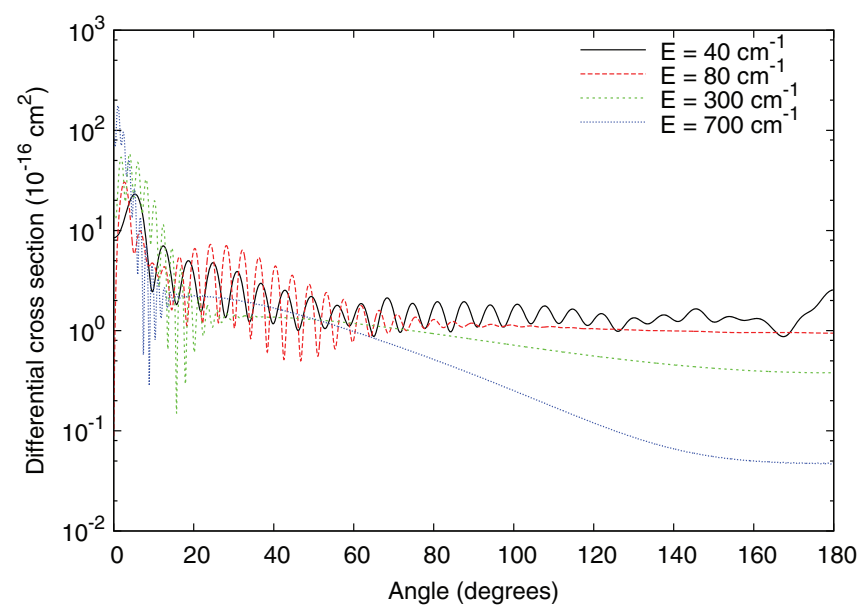

FIG. 8. Inelastic differential cross section $j=0 \rightarrow j=2$ for several energies, as a function of the scattering angle.

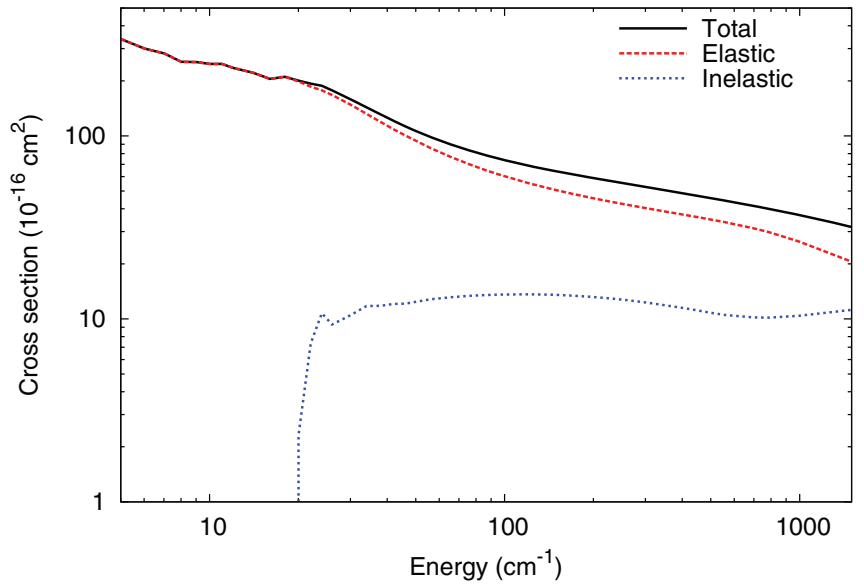

FIG. 9. Momentum transfer cross sections for $j=1$. Full line: total cross section, dashed line: elastic cross section, and dotted line: total inelastic cross section.

the elastic collisions, but the relative contribution of rotational excitation increases with the energy to reach $35 \%$ of the total momentum transfer cross section at $E_{c}=1500 \mathrm{~cm}^{-1}$. The momentum transfer cross sections are generally smaller than the scattering cross sections, and the difference between the two types of cross sections increases with the energy. This effect is caused by the function $\Phi\left(E_{c}, \gamma\right)=1-\sqrt{E_{c}^{\prime} / E_{c}} \cos \gamma$ (see Eq. (14)), which suppresses the contributions from the small angles in the differential cross section. At low energy, we have seen (cf. Figure 7) that the differential cross section can be large for all scattering angles. However, as the energy increases, the contributions from large $\gamma$ decreases rapidly. Therefore, the momentum transfer cross sections decrease more quickly than the scattering cross section as the energy increases.

We found that the total momentum transfer cross sections are similar for all values of $j$ up to a few percent, which can be seen as surprising at high scattering energy. Indeed, we have shown that the inelastic transitions contribute to a large part of the momentum transfer cross section for $j=1$ (see Figure 9), but also that the inelastic cross sections decrease very quickly with increasing initial rotational level (see Figures 5 and 6). We thus expect a lower contribution of inelastic scattering to the momentum transfer cross section as $j$ increases. However, this effect is compensated by the fact that the elastic cross section increases slightly with $j$, as shown in Figure 4. Therefore, we conclude that the total momentum transfer cross section is similar for all initial rotational levels, but that the contribution of the elastic collisions increases with $j$. As an example, at

TABLE III. Total, elastic and inelastic momentum transfer cross sections for $j=0, j=5$, and $j=9$ (in units of $10^{-16} \mathrm{~cm}^{2}$ ) for $E_{c}=128.6 \mathrm{~cm}^{-1}$.

\begin{tabular}{lllr}
\hline \hline & $\sigma_{\text {tot }}^{\mathrm{tr}}$ & $\sigma_{\mathrm{el}}^{\mathrm{tr}}$ & $\sigma_{\text {inel }}^{\mathrm{tr}}$ \\
\hline$j=0$ & 67.5 & 51.0 & 16.5 \\
$j=5$ & 68.0 & 60.5 & 7.5 \\
$j=9$ & 68.0 & 67.4 & 0.6 \\
Ref. 1 & 72 & & \\
\hline
\end{tabular}


TABLE IV. Scattering and momentum transfer rate coefficients for $j=0$ for temperatures in the range 100-300 K. The rates are given in units of $\mathrm{cm}^{3} \mathrm{~s}^{-1}$. Powers of 10 are given in brackets.

\begin{tabular}{|c|c|c|c|c|c|c|}
\hline \multirow[b]{2}{*}{$T(\mathrm{~K})$} & \multicolumn{3}{|c|}{ Scattering rate coefficient, $k(T)$} & \multicolumn{3}{|c|}{ Momentum transfer rate coefficient, $k^{\mathrm{tr}}(T)$} \\
\hline & $k_{\mathrm{el}}$ & $k_{\text {inel }}$ & $k_{\mathrm{tot}}$ & $k_{\mathrm{el}}^{\mathrm{tr}}$ & $k_{\text {inel }}^{\mathrm{tr}}$ & $k_{\mathrm{tot}}^{\mathrm{tr}}$ \\
\hline 100 & $2.36[-9]$ & $8.80[-11]$ & $2.44[-9]$ & $2.70[-10]$ & $6.73[-11]$ & $3.37[-10]$ \\
\hline 125 & $2.53[-9]$ & $9.82[-11]$ & $2.63[-9]$ & $2.70[-10]$ & $7.35[-11]$ & $3.44[-10]$ \\
\hline 150 & $2.70[-9]$ & $1.07[-10]$ & $2.80[-9]$ & $2.72[-10]$ & $7.86[-11]$ & $3.51[-10]$ \\
\hline 165 & $2.79[-9]$ & $1.12[-10]$ & $2.90[-9]$ & $2.74[-10]$ & $8.13[-11]$ & $3.55[-10]$ \\
\hline 175 & $2.85[-9]$ & $1.15[-10]$ & $2.97[-9]$ & $2.75[-10]$ & $8.30[-11]$ & $3.58[-10]$ \\
\hline 185 & $2.92[-9]$ & $1.18[-10]$ & $3.03[-9]$ & $2.76[-10]$ & $8.46[-11]$ & $3.60[-10]$ \\
\hline 195 & $2.98[-9]$ & $1.21[-10]$ & $3.10[-9]$ & $2.77[-10]$ & $8.61[-11]$ & $3.63[-10]$ \\
\hline 205 & $3.03[-9]$ & $1.23[-10]$ & $3.16[-9]$ & $2.78[-10]$ & $8.76[-11]$ & $3.66[-10]$ \\
\hline 250 & $3.28[-9]$ & $1.35[-10]$ & $3.42[-9]$ & $2.83[-10]$ & $9.33[-11]$ & $3.77[-10]$ \\
\hline 300 & $3.52[-9]$ & $1.45[-10]$ & $3.67[-9]$ & $2.89[-10]$ & $9.87[-11]$ & $3.87[-10]$ \\
\hline
\end{tabular}

an energy of $1000 \mathrm{~cm}^{-1}$ the inelastic scattering accounts for about $30 \%$ of the momentum transfer cross section for $j=0$, while this number drops to $13 \%$ for $j=9$.

The energy corresponding to the temperature of the mesospheric sodium, $T=185 \mathrm{~K}$, is $k_{B} T / h c=128.6 \mathrm{~cm}^{-1}$. At this energy, our calculated value for the total momentum transfer cross section is $\sigma^{\text {tr }}=68 \times 10^{-16} \mathrm{~cm}^{2}$ for $j=5$ (the most populated rotational level at $T=185 \mathrm{~K}$ ). We show in Table III the value of the total momentum transfer cross section and the contribution from elastic and inelastic scattering, for three values of $j$. As indicated above, the total cross section is almost independent of $j$, but the contribution from inelastic scattering decreases with $j$. The value estimated by Holzlöhner et al., ${ }^{1}$ who treated $\mathrm{Na}$ and $\mathrm{N}_{2}$ as hard spheres with effective particle radii given by the Van der Waals radii, is $\sigma=72$ $\times 10^{-16} \mathrm{~cm}^{2}$, in surprisingly good agreement given the very simple assumptions made in their calculation. However, more detailed models of the velocity redistribution of the sodium atoms following collisions with $\mathrm{N}_{2}$ can be achieved by using the elastic and inelastic differential cross sections presented in this work over a whole range of energies.

\section{Rates}

The thermal rate coefficient for a transition $j-j^{\prime}$ at a given temperature $T$ is given by the integral of the cross section over a Maxwell-Boltzmann distribution of initial energies:

$$
k_{j j^{\prime}}(T)=\left(\frac{2}{k_{B} T}\right)^{3 / 2} \frac{1}{\sqrt{\pi \mu}} \int_{0}^{\infty} E_{c} e^{-E_{c} / k_{B} T} \sigma_{j j^{\prime}}\left(E_{c}\right) d E_{c} .
$$

The rate coefficient for momentum transfer, $k^{\mathrm{tr}}(T)$, is given by the same expression, replacing $\sigma\left(E_{c}\right)$ by $\sigma^{\operatorname{tr}}\left(E_{c}\right)$.

We compare in Tables IV-VI the rate coefficients for the three initial rotational states $j=0, j=5$, and $j=9$. The elastic rate coefficient is simply $k_{\mathrm{el}}=k_{j j}$, while the inelastic rate coefficient (including excitation and de-excitation transitions) is defined as $k_{\text {inel }}=\sum_{j^{\prime} \neq j} k_{j j^{\prime}}$ and the total rate coefficient is $k_{\text {tot }}=k_{\mathrm{el}}+k_{\text {inel }}$. We only report the rates for temperatures between $100 \mathrm{~K}$ and $300 \mathrm{~K}$, which are close to the average mesospheric temperature, $T=185 \mathrm{~K}$. In this range, the rate coefficients vary slowly with the temperature.

As expected from the previous discussion, the rate coefficient is dominated by the elastic transitions for all initial rotational states. For $j=0$, the ratio between the elastic and inelastic rate is $k_{\mathrm{el}} / k_{\text {inel }} \sim 25$ at $T=185 \mathrm{~K}$, so that inelastic collisions enhance the rate coefficient by less than $5 \%$. The inelastic rate coefficients are dominated by $\Delta j=2$ transitions, as expected from the cross sections of Figure 3 . The total rate coefficients are very similar for the three values of $j$. However, the contribution of inelastic collisions decreases quickly

TABLE V. Same as Table IV, but for the initial rotational state $j=5$.

\begin{tabular}{|c|c|c|c|c|c|c|}
\hline \multirow[b]{2}{*}{$T(\mathrm{~K})$} & \multicolumn{3}{|c|}{ Scattering rate coefficient, $k(T)$} & \multicolumn{3}{|c|}{ Momentum transfer rate coefficient, $k^{\operatorname{tr}}(T)$} \\
\hline & $k_{\mathrm{el}}$ & $k_{\text {inel }}$ & $k_{\mathrm{tot}}$ & $k_{\mathrm{el}}^{\mathrm{tr}}$ & $k_{\text {inel }}^{\text {tr }}$ & $k_{\mathrm{tot}}^{\mathrm{tr}}$ \\
\hline 100 & $2.46[-9]$ & $3.07[-11]$ & $2.49[-9]$ & $3.15[-10]$ & $2.88[-11]$ & $3.44[-10]$ \\
\hline 125 & $2.63[-9]$ & $3.75[-11]$ & $2.67[-9]$ & $3.15[-10]$ & $3.43[-11]$ & $3.49[-10]$ \\
\hline 150 & $2.79[-9]$ & $4.40[-11]$ & $2.83[-9]$ & $3.16[-10]$ & $3.93[-11]$ & $3.55[-10]$ \\
\hline 165 & $2.88[-9]$ & $4.77[-11]$ & $2.93[-9]$ & $3.17[-10]$ & $4.19[-11]$ & $3.59[-10]$ \\
\hline 175 & $2.94[-9]$ & $5.00[-11]$ & $2.99[-9]$ & $3.18[-10]$ & $4.36[-11]$ & $3.62[-10]$ \\
\hline 185 & $3.00[-9]$ & $5.23[-11]$ & $3.06[-9]$ & $3.19[-10]$ & $4.52[-11]$ & $3.64[-10]$ \\
\hline 195 & $3.06[-9]$ & $5.46[-11]$ & $3.12[-9]$ & $3.20[-10]$ & $4.67[-11]$ & $3.67[-10]$ \\
\hline 205 & $3.12[-9]$ & $5.67[-11]$ & $3.18[-9]$ & $3.21[-10]$ & $4.81[-11]$ & $3.69[-10]$ \\
\hline 250 & $3.37[-9]$ & $6.57[-11]$ & $3.43[-9]$ & $3.26[-10]$ & $5.37[-11]$ & $3.79[-10]$ \\
\hline 300 & $3.60[-9]$ & $7.43[-11]$ & $3.68[-9]$ & $3.30[-10]$ & $5.87[-11]$ & $3.89[-10]$ \\
\hline
\end{tabular}


TABLE VI. Same as Table IV, but for the initial rotational state $j=9$.

\begin{tabular}{|c|c|c|c|c|c|c|}
\hline \multirow[b]{2}{*}{$T(\mathrm{~K})$} & \multicolumn{3}{|c|}{ Scattering rate coefficient, $k(T)$} & \multicolumn{3}{|c|}{ Momentum transfer rate coefficient, $k^{\operatorname{tr}}(T)$} \\
\hline & $k_{\mathrm{el}}$ & $k_{\text {inel }}$ & $k_{\text {tot }}$ & $k_{\mathrm{el}}^{\mathrm{tr}}$ & $k_{\text {inel }}^{\mathrm{tr}}$ & $k_{\mathrm{tot}}^{\mathrm{tr}}$ \\
\hline 100 & $2.47[-9]$ & $3.11[-12]$ & $2.47[-9]$ & $3.44[-10]$ & $2.82[-12]$ & $3.46[-10]$ \\
\hline 125 & $2.65[-9]$ & $4.71[-12]$ & $2.65[-9]$ & $3.47[-10]$ & $4.02[-12]$ & $3.51[-10]$ \\
\hline 150 & $2.81[-9]$ & $6.55[-12]$ & $2.82[-9]$ & $3.52[-10]$ & $5.32[-12]$ & $3.57[-10]$ \\
\hline 165 & $2.91[-9]$ & $7.76[-12]$ & $2.92[-9]$ & $3.54[-10]$ & $6.14[-12]$ & $3.61[-10]$ \\
\hline 175 & $2.97[-9]$ & $8.59[-12]$ & $2.98[-9]$ & $3.56[-10]$ & $6.70[-12]$ & $3.63[-10]$ \\
\hline 185 & $3.04[-9]$ & $9.46[-12]$ & $3.05[-9]$ & $3.58[-10]$ & $7.28[-12]$ & $3.65[-10]$ \\
\hline 195 & $3.10[-9]$ & $1.03[-11]$ & $3.11[-9]$ & $3.60[-10]$ & $7.86[-12]$ & $3.68[-10]$ \\
\hline 205 & $3.16[-9]$ & $1.12[-11]$ & $3.17[-9]$ & $3.62[-10]$ & $8.45[-12]$ & $3.70[-10]$ \\
\hline 250 & $3.40[-9]$ & $1.54[-11]$ & $3.42[-9]$ & $3.69[-10]$ & $1.11[-11]$ & $3.80[-10]$ \\
\hline 300 & $3.64[-9]$ & $2.01[-11]$ & $3.66[-9]$ & $3.74[-10]$ & $1.41[-11]$ & $3.88[-10]$ \\
\hline
\end{tabular}

with $j$. The inelastic rate constant for $j=5$ is about a factor of 2 smaller than for $j=0$, while the difference between the rates for $j=9$ and $j=0$ represents an order of magnitude. The ratio between the elastic and inelastic rates thus increases to $k_{\mathrm{el}} / k_{\text {inel }} \sim 60$ for $j=5$ and $k_{\mathrm{el}} / k_{\text {inel }} \sim 300$ for $j=9$. It should also be noted that the ratio decreases with increasing temperature.

For the three values of $j$, the elastic momentum transfer rate coefficient $k_{\mathrm{el}}^{\mathrm{tr}}$ is about an order of magnitude smaller than the elastic scattering rate coefficient $k_{\mathrm{el}}$, while the two types of inelastic rates have a comparable magnitude. In consequence, inelastic transitions play a more important role in the momentum transfer rates. For $j=0$, the ratio between the elastic and inelastic rate is only $k_{\mathrm{el}}^{\mathrm{tr}} / k_{\mathrm{inel}}^{\mathrm{tr}} \sim 3$. The ratio is larger for rotationally excited initial states: for $j=5$, it reaches the value $k_{\mathrm{el}}^{\mathrm{tr}} / k_{\text {inel }}^{\mathrm{tr}} \sim 8$, while we have $k_{\mathrm{el}}^{\mathrm{tr}} / k_{\text {inel }}^{\mathrm{tr}} \sim 50$ for $j=9$.

\section{CONCLUSIONS}

We have performed a complete quantal study of elastic and inelastic rotational collisions of $\mathrm{Na}$ with $\mathrm{N}_{2}$ for collision energies relevant to the modeling of the photon return flux for sodium laser guide stars.

We have obtained the two-dimensional PES of the ground state of the $\mathrm{Na}-\mathrm{N}_{2}$ complex by means of the coupled cluster method implemented in MOLPRO with the $\mathrm{N}-\mathrm{N}$ internuclear distance fixed to its equilibrium geometry. The potential has a minimum at $R=10.47$ a.u. and $\theta=135^{\circ}$. The depth of the well is $26.9 \mathrm{~cm}^{-1}$, and the basis set superposition effect on the depth is about $7 \mathrm{~cm}^{-1}$. The asymptotic part of the potential was constructed analytically by calculating the $C_{6}$ dispersion coefficient.

We investigated the $\mathrm{Na}-\mathrm{N}_{2}$ collisions with the quantummechanical close-coupling method for energies in the range $5-1500 \mathrm{~cm}^{-1}$. We obtained the cross sections for elastic and inelastic rotational scattering. We found that the collisions were dominated by elastic scattering and were largely independent of the initial rotational state. On the other hand, inelastic cross sections were found to decrease quickly with the increase in the initial rotational state. With the differential cross sections, we computed the momentum transfer cross sections. We found the momentum transfer cross sections to be smaller than the scattering cross sections, the difference between the two types of cross sections increasing with the energy of collision. The contribution of inelastic transitions was found to be more important for momentum transfer cross sections than for scattering cross sections. Finally, we calculated the elastic and inelastic rate coefficients for the process for temperatures close to $185 \mathrm{~K}$.

The 2D potential energy surface, the first five radial functions $V_{\lambda}$, as well as the integral and diffusion cross sections presented in this work are available online as Supplementary Material. ${ }^{26}$

\section{ACKNOWLEDGMENTS}

We thank D. Budker, R. Holzlöhner and their collaborators for useful discussions. J.L. is supported by a fellowship from the Belgian American Educational Foundation. The research of A.D. and P.Z. is supported by the Chemical Science, Geoscience, and Bioscience Division of the Office of Basic Energy Science, Office of Science, U.S. Department of Energy.

${ }^{1}$ R. Holzlöhner, S. M. Rochester, D. Bonaccini Calia, D. Budker, J. M. Higbie, and W. Hackenberg, Astron. Astrophys. 510, A20 (2010).

${ }^{2}$ J. M. C. Plane, Atmos. Chem. Phys. 4, 627 (2004).

${ }^{3}$ E. P. F. Lee and T. G. Wright, Chem. Phys. Lett. 392, 187 (2004).

${ }^{4}$ P. J. Knowles, C. Hampel, and H.-J. Werner, J. Chem. Phys. 99, 5219 (1993)

${ }^{5}$ J. D. Watts, J. Gauss, and R. J. Bartlett, J. Chem. Phys. 98, 8718 (1993).

${ }^{6}$ H.-J. Werner, P. J. Knowles, R. Lindh, F. R. Manby, M. Schütz, et al., MOLPRO, version 2009.1, a package of ab initio programs, 2009, see http://www.molpro.net.

${ }^{7}$ T. H. Dunning, Jr., J. Chem. Phys. 90, 1007 (1989); B. P. Prascher, D. E. Woon, K. A. Peterson, T. H. Dunning, Jr., and A. K. Wilson, Theor. Chem. Acc. 128, 69 (2011).

${ }^{8}$ S. F. Boys and F. Bernardi, Mol. Phys. 19, 553 (1970).

${ }^{9}$ A. J. Stone, The Theory of Intermolecular Forces, (Clarendon, Oxford, 1997).

${ }^{10}$ D. M. Bishop and J. Pipin, J. Chem. Phys. 97, 3375 (1992).

${ }^{11}$ R. Moszynski, P. S. Zuchowski, and B. Jezioski, Collect. Czech. Chem. Commun. 70, 1109 (2005); T. Korona, M. Przybytek, and B. Jeziorski, Mol. Phys. 104, 2303 (2006).

${ }^{12}$ A. K. Wilson, T. v. Mourik, and T. H. Dunning, Jr., J. Mol. Struct.: THEOCHEM 388, 339 (1996).

${ }^{13}$ W. J. Meath and A. Kumar, Int. J. Quantum Chem. 24, 501 (1990); O. Christiansen, A. Halkier, H. Koch, P. Jøgensen, and T. Helgaker, J. Chem. Phys. 108, 2801 (1998); G. Maroulis, ibid. 118, 2673 (1998).

${ }^{14}$ A. Derevianko, S. G. Porsev, and J. F. Babb, At. Data Nucl. Data Tables 96, 323 (2010). 
${ }^{15}$ A. M. Arthurs and A. Dalgarno, Proc. R. Soc. London, Ser. A 256, 540 (1960).

${ }^{16}$ D. R. Flower, Molecular Collisions in the Interstellar Medium, 2nd ed. (Cambridge University Press, Cambridge, 2007).

${ }^{17}$ J. Bendtsen, J. Raman Spectrosc. 2, 133 (1974).

${ }^{18}$ A. Lothus and P. H. Krupenie, J. Phys. Chem. Ref. Data 6, 113 (1977).

${ }^{19}$ J. M. Hutson and S. Green, MOLSCAT, computer code, version 14 (1994), distributed by the Collaborative Computational Project No. 6 of the Engineering and Physical Sciences Research Council (UK).

${ }^{20}$ G. A. Parker and R. T. Pack, J. Chem. Phys. 68, 1585 (1978).
${ }^{21}$ P. G. Burke, D. Scrutton, J. H. Tait, and A. J. Taylor, J. Phys. B 2, 1155 (1969).

${ }^{22}$ J. T. Muckerman and R. B. Bernstein, Chem. Phys. Lett. 4, 183 (1969).

${ }^{23}$ L. N. Smith, D. J. Malik, and D. Secrest, J. Chem. Phys. 71, 4502 (1979).

${ }^{24}$ D. C. Clary, J. N. L. Connor, and H. Sun, Mol. Phys. 49, 1139 (1983); V. Aquilanti, L. Beneventi, G. Grossi, and F. Vecchiocatti, J. Chem. Phys. 89, 751 (1988).

${ }^{25}$ S. I. Chu and A. Dalgarno, Proc. R. Soc. London, Ser. A 342, 191 (1975); R. Goldfalm, D. J. Kouri, and S. Green, J. Chem. Phys. 67, 5661 (1977).

${ }^{26}$ See supplementary material at http://dx.doi.org/10.1063/1.3653983 for the potential and cross sections. 